\title{
Wireless Sensors and Networks for Advanced Energy Management
}

\author{
James E. Hardy and Wayne W. Manges, Oak Ridge National Laboratory \\ Jose A. Gutierrez, Eaton Corporation \\ Phani Teja V. Kuruganti, University of Tennessee
}

\begin{abstract}
Numerous national studies and working groups have identified low-cost, very low-power wireless sensors and networks as a critical enabling technology for increasing energy efficiency, reducing waste, and optimizing processes. Research areas for developing such sensor and network platforms include microsensor arrays, ultra-low power electronics and signal conditioning, data/control transceivers, and robust wireless networks. A review of some of the research in the following areas will be discussed:

- Low-cost, flexible multi-sensor array platforms $\left(\mathrm{CO}_{2}, \mathrm{NO}_{\mathrm{x}}, \mathrm{CO}\right.$, humidity, $\mathrm{NH}_{3}, \mathrm{O}_{2}$, occupancy, etc.) that enable energy and emission reductions in applications such as buildings and manufacturing

- Modeling investments (energy usage and savings to drive capital investment decisions) and estimated uptime improvements through pervasive gathering of equipment and process health data and its effects on energy

- Robust, self-configuring wireless sensor networks for energy management

- Quality-of-service for secure and reliable data transmission from widely distributed sensors

Wireless communications is poised to support technical innovations in the industrial community, with widespread use of wireless sensors forecasted to improve manufacturing production and energy efficiency and reduce emissions. Progress being made in wireless system components, as described in this paper, is helping bring these projected improvements to reality.
\end{abstract}

\section{Introduction}

Numerous national studies and working groups have identified low-cost, very low-power wireless sensors and networks as a critical enabling technology for increasing energy efficiency, reducing waste, and optimizing processes. A Department of Energy report (DOE 2002) forecasted that the widespread use of wireless sensors will improve manufacturing production and energy efficiency by $10 \%$ and reduce emissions by $25 \%$. An example of this is that almost one-quarter of all the electricity consumed in the United States comes from motor-driven systems. Implementation of advanced energy management monitoring and diagnostic systems in large electric motor (>200 HP) applications has shown dramatic energy savings, economic benefits, and reduced environmental impact. Monitoring of critical data from medium to small motors faces a technological barrier due to the lack of a robust, secure, and cost-effective communications network; the use of conventional field wiring practices is prohibitively expensive and lacks scalability. Cost-effective, robust wireless sensors and networks are the solution. To achieve these solutions, low-cost sensors and computing are necessary as well as robust, reliable wireless communications. Low-cost sensors are being developed using microsensor (MS) technology. Based on semiconductor or semiconductor-like processes, MS 
arrays are designed and fabricated at very low cost (in high volumes). The low cost aspect will allowed sensors to be distributed throughout processes to aid in more and better monitoring which will lead to improved process control, system uptime, enhanced yield, and emission reductions. For a cost-effective implementation of these MS arrays, wireless communications/networks are essential. Installing wires to all of the new sensors is cost prohibitive, wireless technology as well as open/standard interfaces for interoperability are also requirements. Finally, for acceptance in the manufacturing world, the quality-of-service (throughput, latency, reliability, security, etc.) must be high. A brief description will be given for each of these research areas and challenges to be addressed will be described.

\section{Microsensor Array Platforms}

Research at Oak Ridge National Laboratory (ORNL) has been addressing the development of an ultra low-power, low-cost sensor platform based on MS arrays that also include very low-power electronics, signal processing, and two-way data communications, all integrated into a single package (Lavrik 2004; Warmack \& Hardy 2005). MSs were developed to measure carbon dioxide and room occupancy. Advances were made in coatings for detecting carbon dioxide (important for indoor air quality), sensing thermal energy with MSs (for use as a room occupancy detector), and capacitive, very low-power electronics (each of which will be discussed here in turn). An integrated platform was realized that included gas sensing, temperature, humidity, and room occupancy with on-board communications. This work has helped move the state-of-the-art in low-cost wireless sensors closer to implementation. Various aspects of the research are described.

MS sensors coated with a sol-gel mixture responded to $\mathrm{CO}_{2}$ (Ishihara et al. 1993; Lang, Wiemhofer, \& Gopel 1996) with a decrease in capacitance, while capacitance increased with humidity pulses. This is explained by the change in polarity of the interacting dielectric material. Unfortunately, the performance of the coated sensors was more variable than desired (Roth et al. 1996). The material was also subject to degradation due to the presence of species such as $\mathrm{SO}_{2}$ and $\mathrm{NO}_{2}$, though temperature cycling appears to mitigate oxidization and extend the useful lifetime. The sensitivity of the MEMS sensors is more than adequate for sensing $\mathrm{CO}_{2}$ for ventilation purposes (Yamazoe et al. 1996), but more work is needed to produce reproducible sensors; i.e. long-term drift and coating degradation are still issues. Figure 1 shows the response of an MS to various $\mathrm{CO}_{2}$ levels. The MS response tracks the $\mathrm{CO}_{2}$ impulses very closely and responds over a wide range of concentrations, from $3000 \mathrm{ppm}$ to $100 \mathrm{ppm}$, that easily covers the range of indoor air quality concerns (300 ppm to $1000 \mathrm{ppm}$ ). The zero-drift, as seen illustrated in Figure 1 by the Delta-C/C (change in capacitance divided by the capacitance) response not returning to zero in the later pulses, is a common problem with MS technology. Elevating the MS base temperature to $70^{\circ} \mathrm{C}$ improves stability but further development in stable coatings is needed before these MS arrays can be fielded. 


\section{Figure 1. MS output response to various $\mathrm{CO}_{2}$ input levels}

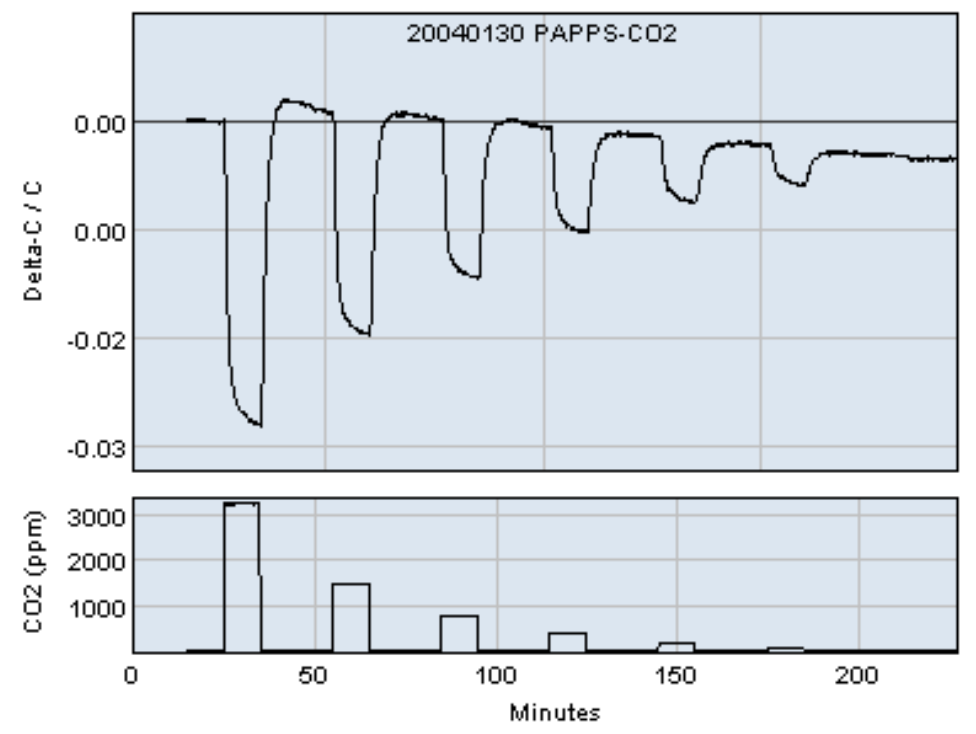

A first-of-a-kind, very low power, low-cost MS was also developed by ORNL to detect thermal energy. These infrared (IR) detectors were suspended, bimaterial microcantilevers made from polysilicon with a gold layer as the bimaterial layer (Datskos, Lavrik, and Rajic 2004). The cantilever detectors were capacitively read using a custom very low power analog application specific integrated circuit (ASIC.) The noise level was about $10^{-16}$ farads at a readout rate of 10 $\mathrm{KHz}$. The detectors were designed to operate under ambient conditions. A measure of detector performance is the noise equivalent temperature difference (NETD). In order for a detector to be able to detect heat from a human, a value of NETD of $500 \mathrm{mK}$ or lower is required. The predicted NETD for the designed detectors was about $20 \mathrm{mK}$ in air and less than $5 \mathrm{mK}$ in vacuum.

In actual tests these detectors responded to a soldering iron (temperatures $>150 \mathrm{C}$ ). We expect to achieve detection of room temperature objects by increasing the detector area and eliminating thermo-mechanical noise sources. Designs with modified geometry and new materials have been considered and will be fabricated in the near future. For the first implementation of a MS IR detector using low-power capacitive readout, the results were very encouraging and the modified designs should produce a useable detector for room occupancy.

The readout and conditioning electronics for the MS system were designed to be operated on ultra-low power ( $<1 \mathrm{~mW}$ continuous operation or $\sim 100 \mu \mathrm{W}$ in sample mode) (Britton et al. 2000, 2001). Hardware and software were developed to read various sensors that detect room environmental conditions. The hardware consisted of a circuit board set containing the readout circuit (custom ASIC), a microcontroller to communicate with the host computer via an AC line coupler, and various sensors. The circuit board set also included a daughterboard that contained the MS sensor array (10 individual MSs) and the ASIC and a motherboard that contained the remaining circuitry. The ASIC is a mixed-mode capacitive readout chip with 10 inputs and is fully programmable via a 4-wire SPI interface. The ASIC also contains a thermometer circuit for sensing chip temperature. The occupancy detection circuit, a temperature and humidity sensor, and the MS array heater control electronics are on the motherboard. The system is capable of reading any of the MSs, their temperature, the ASIC temperature, ambient temperature \& 
humidity, and any room movement since the last reading. It also has the ability to heat the MS array. The ASIC can be connected to wireless communication circuitry or to communication systems that send data over existing power lines The integrated sensor, electronics, and data transmission package is shown in Figure 2, which can measure $\mathrm{CO}_{2}$, room occupancy, humidity, and temperature.

Figure 2. Integrated MS array with ultra-low power electronics

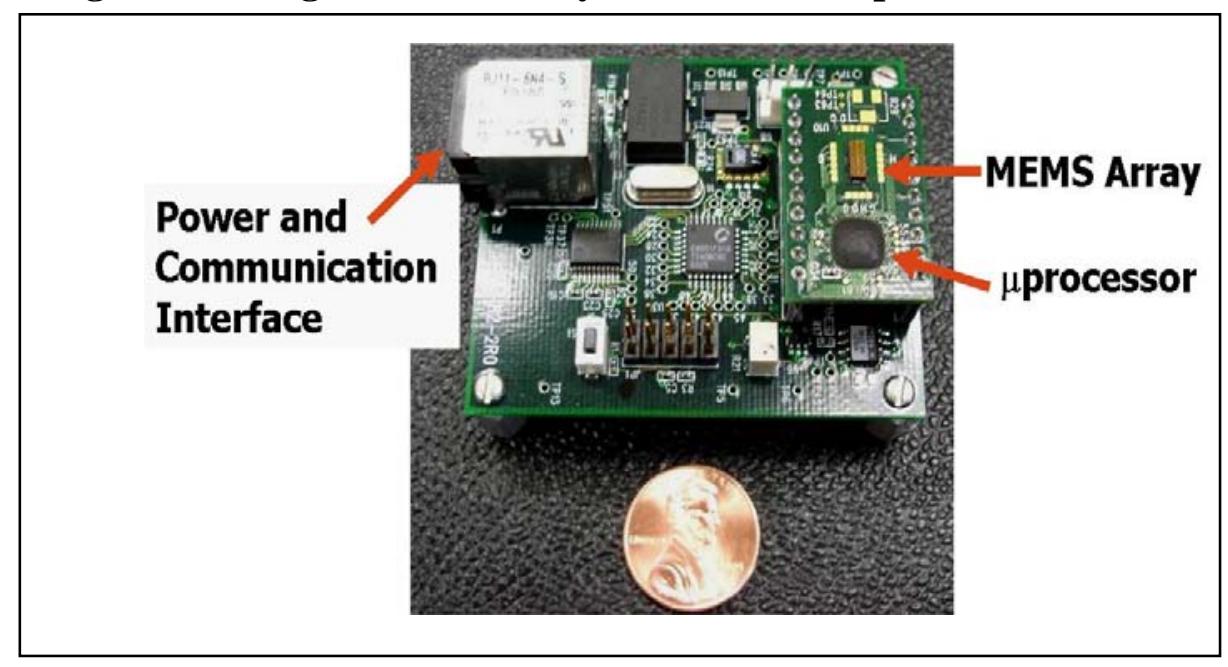

Two significant application areas for this MS platform are in the buildings area and in motor control (MS and wireless networks are applicable in numerous areas; buildings will be described briefly and motor control in more detail.)

Building Automation Systems (BASs) are often set to bring in outside air to ventilate indoor rooms on a routine basis and by a set amount (15 cfm/person). While helping to ensure the indoor air quality (IAQ) is maintained, this practice can be in direct opposition to energy savings. By measuring $\mathrm{CO}_{2}$, humidity, occupancy, and temperature in each conditioned space, more intelligent ventilation and energy savings strategies can be employed. Ventilation can be done on demand rather than on a routine cycle. Estimates from several studies (Fehlmann et al. 1993; Gabel et al. 1986; Zamboni et al. 1991) indicate a potential energy savings of 20-30\% while maintaining and perhaps even improving the IAQ. First order estimates indicate the potential reduction of energy consumption (if deployed in most U.S. buildings) could be 2 quads, reducing energy costs by $\$ 55$ billion and carbon emissions by 35 million metric tons (Orme, $\mathrm{M}$. 1998). Additionally, improved IAQ can increase productivity and reduce health care costs substantially, resulting in over $\$ 100$ Billion in savings a year (Vaughn 2001). These benefits can only be achieved by employing a whole-building perspective that integrates these sensors with feedback to the BAS controllers that can respond dynamically and that can optimize the system for energy efficiency and IAQ.

\section{Closing the Loop on Energy Savings with Wireless Communications}

According to Department of Energy (DOE) research (Xenergy. 1998), motors below 200 horsepower make up $98 \%$ of the motors in service and consume $85 \%$ of the energy used; therefore widespread deployment of energy management systems is critical in realizing the 
possible savings. This energy savings application is similar to buildings in the need for affordable sensors and energy management systems. Wireless sensor technology enables existing and new electrical distribution and power control systems to communicate and report information for energy management as well as for diagnostic and prognostic purposes. Today, only a small percentage of this equipment is capable of communicating information due to cost of installation and the use of proprietary sensor interfaces that lack interoperability. Wireless Industrial Sensor Networks (WISN) provide a low-cost path to deliver voltage, current, power, load, and other key process information to facility/enterprise systems or applications. WISN will enable these systems to save energy, provide diagnostics and prognostic capabilities, and improve uptime across the entire plant. Eaton is currently installing a beta WISN at the BP Whiting Refineries in Indiana. The WISN will help enable on-line estimation of motor efficiency and power usage. Understanding how the energy is being used throughout the power distribution system is the first step in realizing these energy savings. Due to the high cost of installing monitoring equipment today, companies are restricted to deploying only limited, temporary instrumentation to make any assessments. Despite this, the information collected has proven to be helpful in optimizing power distribution systems. WISN will enable a step change in energy management solutions by allowing widespread implementation and continuous, distributed monitoring of plant components. Figure 3 illustrates four models to achieve the energy savings vision using WISNs.

Figure 3. Closing the loop on energy savings

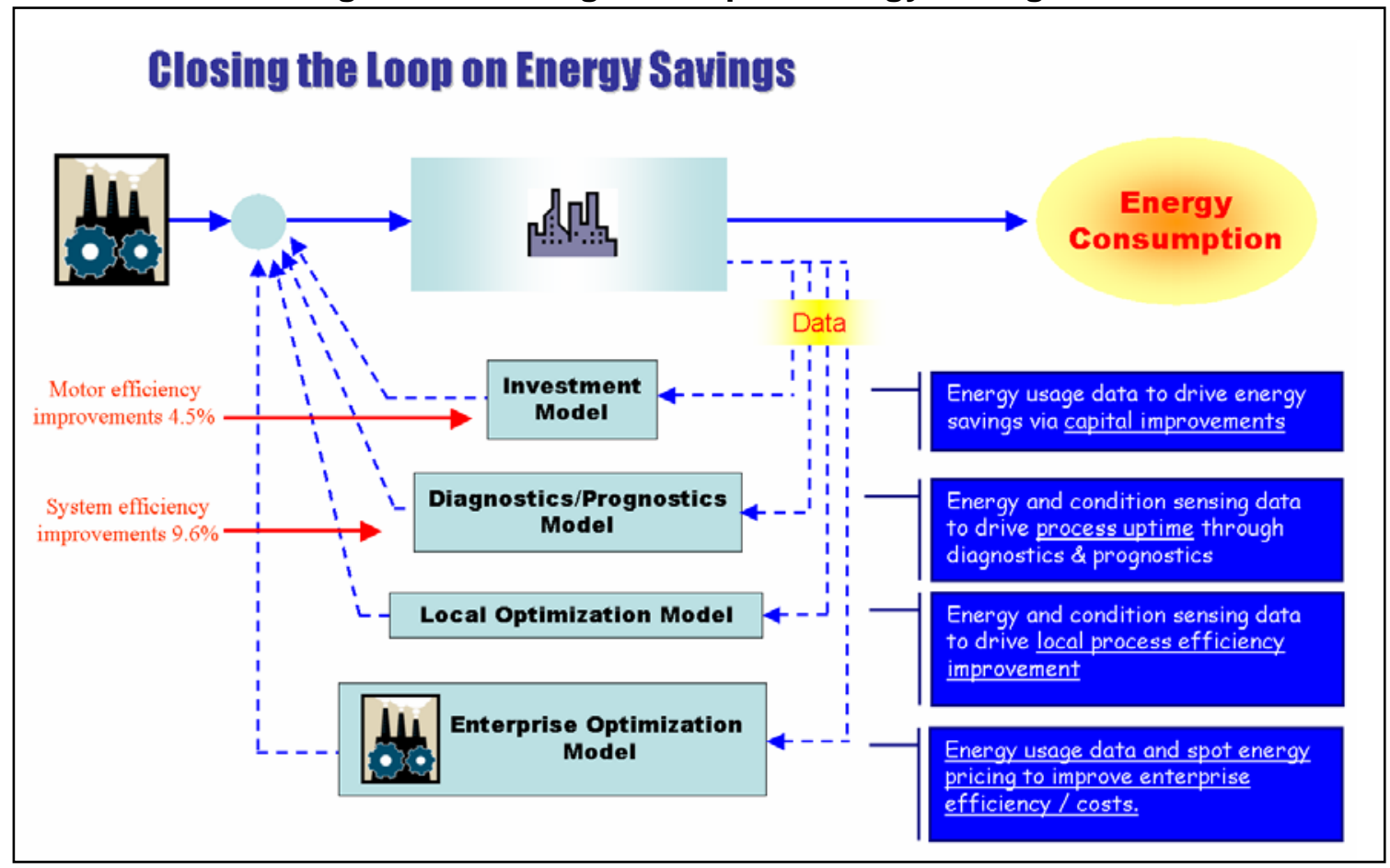

- Investment Model: This is the most basic model and it is focused in using the energy usage data gathered through the energy acquisition system to drive capital investment decisions. At design time, all equipment planned for a particular plant is sized for a particular operating point based on capacity/utilization projections. If these projections are not accurate during actual operation, the operating point of these loads will be likely to cause excess energy consumption. This is typical of motor installations; these motors 
are very efficient while functioning at the designed operational load, but are less efficient at non-design loads. Uncovering the energy usage data for each load will help drive capital investment decisions to lower energy usage (help size future motor installations, help determine motor usage profiles, etc.).

- Diagnostic/Prognostic Model: Maintaining production and avoiding downtime is the primary goal of motor-system monitoring. Energy savings rank below this goal and are thus often neglected. By enabling the pervasive gathering of process data to drive process uptime and merging the energy data as part of a diagnostic/prognostic system, a plant manager will achieve his most important goals while keeping an eye on the energy consumption since it is now part of the process.

- Local Optimization Model: This model is centered in the utilization of energy and information management to drive local process efficiency improvements and to lower overall energy consumption and cost by scheduling these processes to avoid peak energy charges.

- Enterprise Optimization Model: This final model merges the energy usage data and spot energy pricing to improve overall enterprise energy management and lower cost (avoiding peak demand charges, taking advantage of lower price electricity, etc.).

The focus of current efforts is centered on the first two models; the success of these will pave the way for the local and enterprise optimization models since they represent incremental changes to the overall system.

\section{Robust, Self-configuring Wireless Industrial Sensor Networks for Energy Management}

As indicated above, to achieve improvements on a broad scale, energy management solutions need to be extended widely across the energy distribution systems within the process plant. A key barrier in realizing this vision is a robust, secure, and cost-effective communication network that facilitates collection of this critical monitoring and diagnostic information. Today, the cost of communication networks using conventional field wiring practices is prohibitively expensive. To address this problem wireless networks are being researched to reduce communication installation and infrastructure costs by up to $84 \%$ (President's Committee of Advisors on Science and Technology. 1997).

To accomplish this vision, a WISN needs to:

1) Operate with a variety of open, wireless protocols,

2) Self-configure a network that will operate robustly and securely in difficult industrial environments, and

3) Integrate the needed power system sensors with the advanced energy management system software.

Three areas of research must be completed in order to monitor and optimize the electrical power consumption within an industrial process plant:

- Smart sensors with embedded intelligence must be developed to measure parameters, such as, voltage, current, and temperature for a significantly reduced installed cost. 
-Wireless networking must be robust, secure, non-proprietary, self-configuring and costeffective.

- Advanced model-based energy management, diagnostic and control systems must take into account the full complexity of the plants being monitored.

A key enabler for WISN technology is the emerging Low-Rate Wireless Personal Area Network (LR-WPAN) technology instantiated in the IEEE 802.15.4 standard. LRWPAN/IEEE802.15.4 is an open architecture wireless network protocol specifically designed for low-cost and very low-power wireless communications. This technology is a good choice for WISNs focused on monitoring and diagnostic applications; other wireless technologies have focused on communications with higher data throughput and enhanced quality of service e.g. Wireless Local Area Network (WLAN) and other consumer electronics applications. Figure 4 represents the tiers of standard wireless solutions available for various types of networking applications.

Figure 4. Standard Wireless Networking Technologies

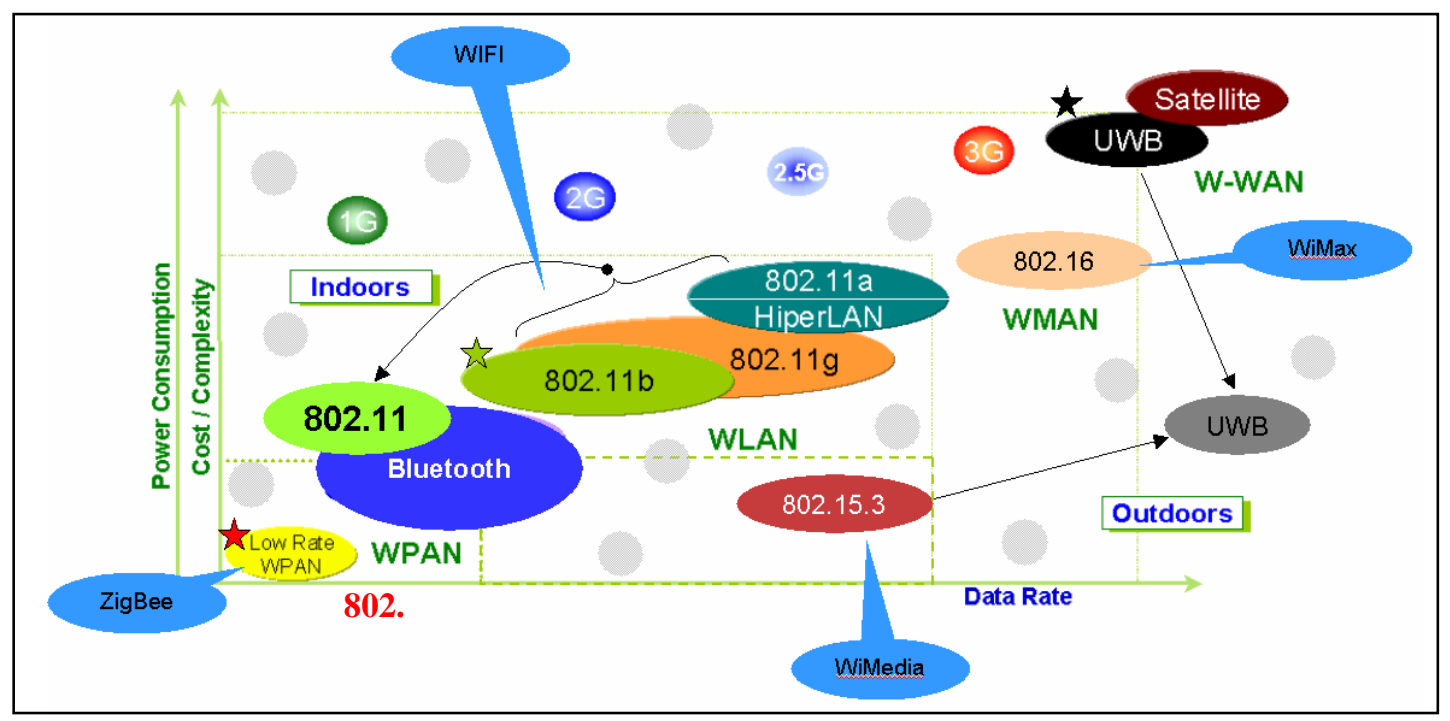

The LR-WPAN design enables wireless communications for sensors and actuators where minimal bandwidth utilization is required. Unlike WLANs, LR-WPAN was designed to convey information without the use of pre-established infrastructure. Self-configuration, multi-path and multi-hop are key attributes of LR-WPAN that enable large scale, mesh type networks to be formed to cover long distances and provide redundant paths within large facilities. LR-WPAN is ideally suited to these types of monitoring and diagnostic applications where the nondeterministic nature of the sensor update rates do not cause control issues. Figure 5 illustrates how the self-configuring mesh network is able to form flexible communications paths between nodes that overcome issues associated with the conventional star network topography. 
Figure 5. Self-configurating mesh network with redundant communications vs. fixed star topology

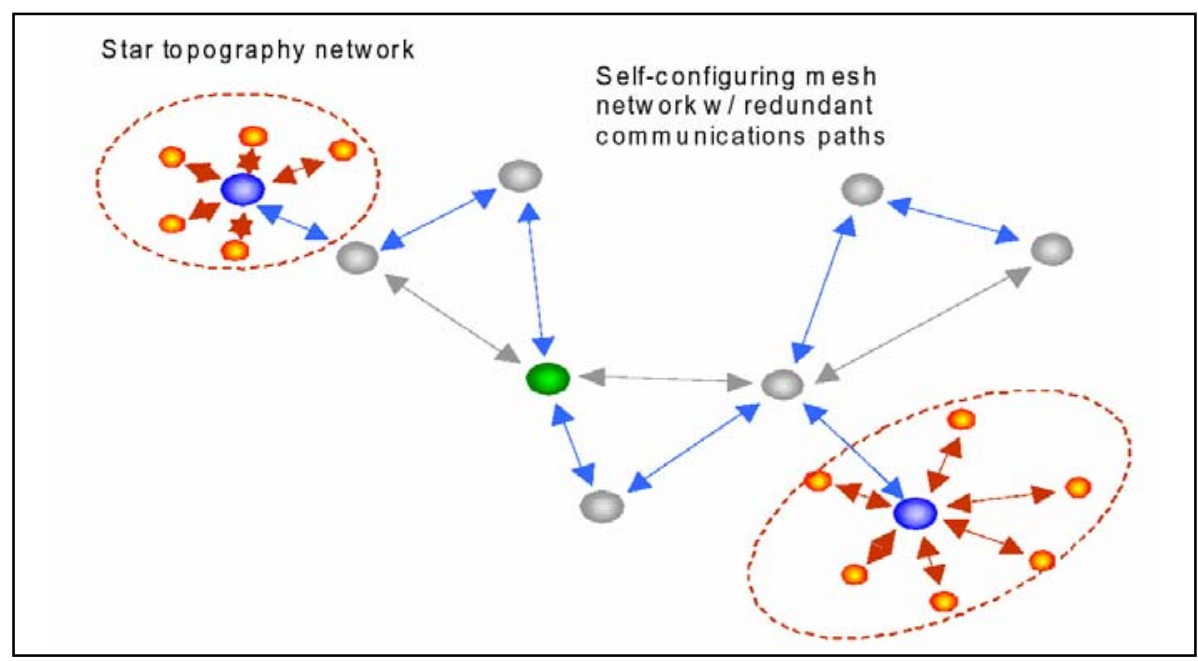

\section{Quality-of-Service for Secure and Reliable Data Transmission from Widely Distributed Sensors}

Wireless technology is fueling new paradigms in personal, commercial, and industrial communications systems throughout the marketplace. With the rapid growth in the networked environments for different industrial and scientific applications, there is a vital need to assure the user or application a certain level of Quality of Service (QoS) in performing a particular transaction over the network. For example, if an industrial site incorporates a wireless distributed control of certain processing equipment or networked control of robots in the manufacturing plant, the QoS of the network carrying the control packets define the functionality, performance or energy efficiency of the industrial process. A Measure of Effectiveness (MOEs), in this case QoS, provides a means for evaluating the overall effectiveness (i.e. energy trade-off analysis) of a proposed wireless communication design while the Measures of Performances (MOPs) throughput, latency, reliability, security, adaptability, and affordability - represent the aggregate properties that define QoS. The MOPs/MOEs are based on mapping functional needs onto operational requirements and defining measurable and quantifiable parameters. Details concerning QoS and its associated MOPs in a WISN are as follows:

\section{Throughput}

Addresses the ability of the network to carry the offered traffic by the industrial applications based on the WISN implementation and as impacted by the communication environment.

\section{Latency}

Addresses the timing considerations for the application information to be carried by the WISN implementation including the characterization of the time delay as well as the variation in the time delay, i.e., jitter. Latency requirements for certain communication traffic, such as a machine's servo control sensors, will have very strict requirements while a WISN to support 
supply chain management might have a more relaxed latency requirement. Factors influencing latency include sensor node density, interrogation rates, network topology, and number of simultaneous actions.

\section{Reliability}

Addresses the ability of the network to carry out its functional requirement of carrying industrial application information over the network over a broad range of operational conditions. Issues include the ability of the network to address communication disruptions, unanticipated variations in traffic and variations in the operational environment using a predictable "fail-safe" mechanism. In addition, WISN are often energy-constrained and therefore reliability includes the ability of the network to maintain operational integrity under energy constraints such as dirty power, battery operation, or energy scavenging.

\section{Security}

Addresses the operational impact and cost associated with the failure to prevent typically three categories of attacks on the communication network: (1) unauthorized interception of confidential information, (2) modification of information and network control messages and (3) interruption of information and network control messages (Perrig, Stankovic, \& Wagner 2004). WISN deployed in an industrial environment can be subject to both physical and logical security attacks. A deep concern to industrial companies is maintaining confidentiality of trade practices. Utilizing wireless technology can appear to be a potential compromise of this requirement; especially, if the information carried on the wireless devices can be used to divulge information pertaining to an industrial process. Therefore, the industrial network's capability to prevent and detect unauthorized interception needs to be evaluated. In addition, industrial networks could be subject to malicious attacks that would compromise integrity of information, as well as the availability of sensor network functions. The capability to handle malicious attacks involving modification and interruption of data and network control messages needs to be addressed in designing the industrial network. The assessment process needs to balance the tradeoff between the security requirements of the industrial communication network with the communication administration overhead associated with security protocols, i.e., security implementation cost needs to be balanced against the other QoS cost performance constraints.

\section{Adaptability}

Central to WISN is its ability to adapt to new reconfigurations and new tasks. Adaptations required by the industrial application include mobility in all or some of the sensor elements as well as scaling and/or reconfiguring the WISN to handle a new process or modification to an existing process as a result of new tasking or goals. The WISN will also need to adapt to handle variations in the industrial environment over the time frame of seconds to years, e.g., RF propagation effects due to dynamic changes in stock supplies or retooling a plant's manufacturing line. The wireless network needs to therefore be capable of handling variations in the traffic flow due to changes in sensor location and machine or process utilization. The network will need to adapt to changes in the environment while maintaining the required levels of throughput, latency, reliability and security. In addition, if the WISN is too complex to adapt and reconfigure to changing needs and a changing environment, then an inherent advantage to 
the WISN approach is compromised. To fully enable WISN, the adaptation of the wireless network should be transparent to the end user. Within the industrial environment, adaptability is one true advantage of wireless over wired networks. Directly interconnecting all neighboring nodes with wiring is too complex and cost prohibitive. A WISN, however, provides a low cost, redundant connectivity that can be exploited in the network layer to optimize routing for current conditions.

\section{Affordability}

Once functionality, operability, and utility are addressed, there will be a need to assess affordability. Affordability in this context includes: cost of ownership (packaging requirements, modifications, maintainability, etc.), implementation costs, replacement and logistics costs, and training and servicing costs as well as the per unit costs.

To address these challenges, a design framework for developing WISN is proposed as illustrated in Figure 6.

\section{Figure 6. Design framework for developing WISN for industrial \& manufacturing} applications.

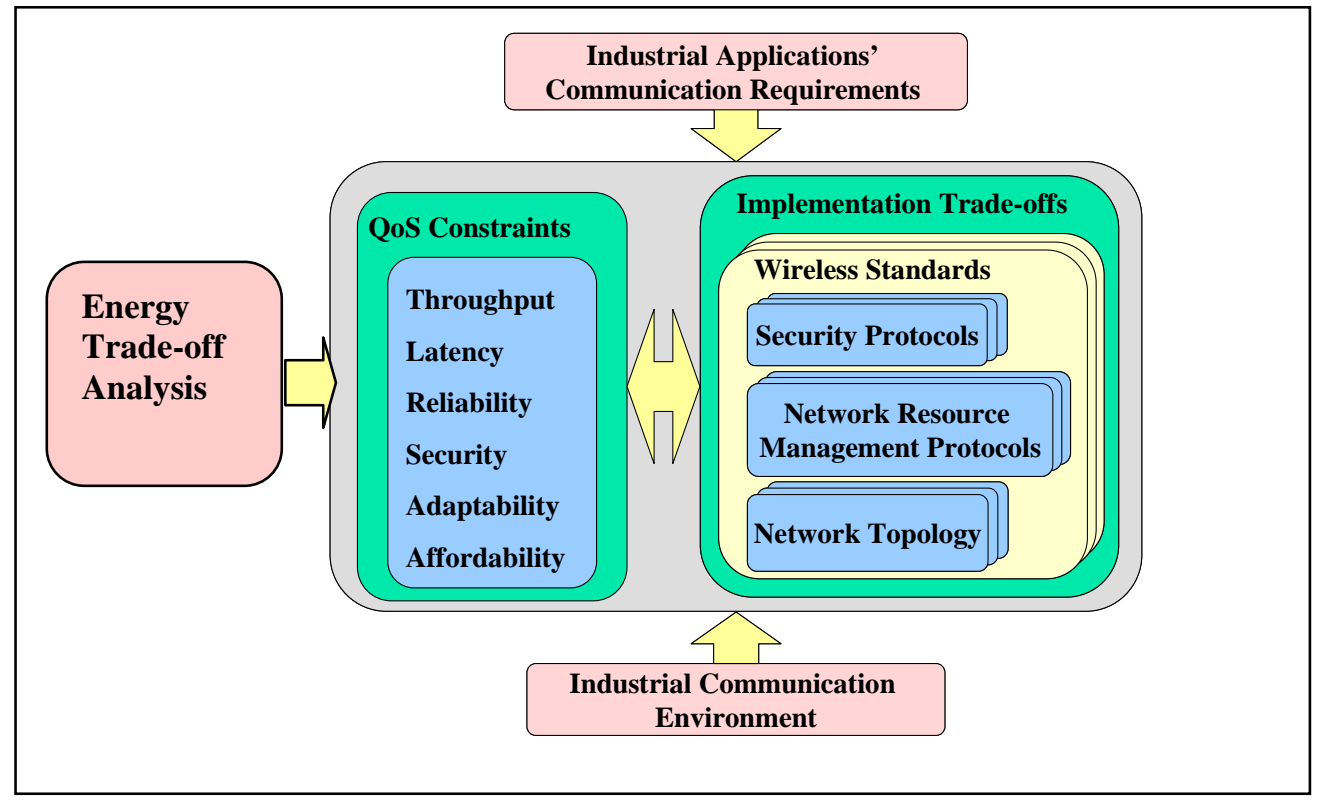

\section{Summary}

Significant energy savings and emission reductions are aided through the addition of more sensors and controls into industrial processes. To make these additions and the potential savings and reductions reality, low-cost, low-power, wireless sensors and networks are critical. WISNs are needed to assist in acquiring data, transforming the data into information and knowledge, and using that information/knowledge to control processes and entire plants. Thus, WISNs can enable energy savings, diagnostics, prognostics, and waste reductions and improve the uptime of the entire plant. To achieve these goals, the WISNs must be reliable, adaptable, affordable, and robust. Research is underway in several areas to move WISNs to the factory 
floor. Platforms for low-cost, low-power, wireless microsensor arrays have been prototyped and robust, self-configuring wireless networks are in development and early deployment. Additionally models of energy management and economics (investment, diagnostic/prognostic, optimization, and enterprises) are being generated, and quality-of-service and performance are being defined. Low-cost sensors and wireless networks will enable widespread implementation of distributed information gathering. This will lead to improved energy efficiencies and production as well as minimizing waste and emissions as evidence by the DOE study that estimated increases in efficiency of $10 \%$ and reductions in emissions by $25 \%$.

\section{References}

Britton, C.L., R.L. Jones, P.I. Oden, Z. Hu, R.J. Warmack, S.F. Smith, W.L. Bryan, and J.M. Rochelle. 2000. “Multiple-input microcantilever sensors”. Ultramicroscopy, 82(1-4): 1721.

Britton, C.L., R.J. Warmack, W.L. Bryan, P.I. Oden, T.G. Thundat, and R.L. Jones. 2001. A Capacitively Readout Multielement Sensor Array with Common-Mode Cancellation. U.S. Patent No. 6,167,748.

Datskos, P. G., N. V. Lavrik, and S. Rajic. 2004. “Microcantilever thermal detector.” Review of Scientific Instruments 75: 1134.

[DOE] Department of Energy. 2002 Industrial Wireless Technology for the $21^{\text {st }}$ Century, Washington, D.C.: U.S. Department of Energy, Office of Energy Efficiency and Renewable Energy Report, Available online: http://www.eere.energy.gov/industry/sensors_automation/pdfs/wireless_technology.pdf. Washington, D.C.: US Department of Energy.

Fehlmann, J., H. Wanner, and M. Zamboni. 1993. „Indoor air quality and energy consumption with demand controlled ventilation in an auditorium." Proceedings of the $6^{\text {th }}$ International Conference on Indoor Air Quality and Climate 5: 45-50.

Gabel, S.D., J. E. Janssen, J.O. Christoffel, and S. E. Scarborough. 1986. “Carbon dioxide-based ventilation control system demonstration.” DE-AC79-84BP15102. Washington, D.C.: U.S. Department of Energy.

Ishihara, T. and K. Kometani. 1993. "Capacitive-Type Gas Sensor for the Selective Detection of Carbon-Dioxide”. Sensors. Actuator B-Chem 13(1-3): 470-472.

Lang, T., H.D. Wiemhofer and W. Gopel. 1996. "Carbonate based CO2 sensors with high performance.” Sensors. Actuator B-Chem. 34(1-3): 383-387.

Lavrik, N. V., M. J. Sepaniak, \& P. G. Datskos. 2004. “Cantilever transducers as a platform for chemical and biological sensors.” Review of Scientific Instruments 75: 2229

Orme, M. 1998. Energy impact of ventilation. Technical Note 49, International Energy Agency Air Infiltration and Ventilation Centre, Coventry, Great Britain. 
Perrig A., J. Stankovic, \& D. Wagner. 2004 “Security in wireless sensor networks,” Communications of the ACM 47(6): 53-57.

President's Committee of Advisors on Science and Technology, Energy Research and Development Panel 1997. Federal energy research and development for the challenges of the 21st century, p. 3-16, Washington D.C.

Roth, M., R. Hartinger, R. Faul and H. E. Endres. 1996. “Drift reduction of organic coated gassensors by temperature modulation.” Sensors. Actuator B-Chem. 36(1-3): 358-362.

Warmack, R. J., and J. E. Hardy. 2005. “Trends in Sensing Technology.” Paper presented at the International Forum Process Analytical Technology Workshop, Washington, D. C., January 12.

Yamazoe, N., et al. 1996. "Gas sensing interfaces of solid electrolyte based carbon dioxide sensors attached with metal carbonate.” Sensors. Actuator B-Chem. 34(1-3): 361-366.

Xenergy, Inc. 1998. United States Industrial Electric Motor Systems Market Opportunities Assessment, Washington, D.C.

Zamboni, M., O. Berchtold, C. Filleux, J. Fehlmann, and F. Drangsholt 1991. “Demand controlled ventilation - An application to auditoria." Proceedings of the $12^{\text {th }}$ AIVC Conference Air Movement \& Ventilation Control within Buildings, pp. 143-155. Coventry, U.K.: Air Infiltration and Ventilation Centre. 